\section{HSE}

Historia Social y de la Educación

Social and Education History
Hipatia Press

www.hipatiapress.com

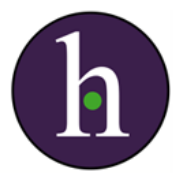

Instructions for authors, subscriptions and further details:

http://hse.hipatiapress.com

\title{
La educación musical en la Institución para la enseñanza de la mujer en Valencia
}

Esperanza Clares-Clares ${ }^{1}$ y Elena Micó-Terol ${ }^{2}$

1) Universidad de Murcia (España)

2) IES Jaume I Ontinyent (Valencia). Generalitat Valenciana

Date of publication: February $23^{\text {th }}, 2019$

Edition period: February 2019-October 2019

To cite this article: Clares-Clares, E. y Micó-Terol, E. (2019) La educación musical en la Institución para la Enseñanza de la Mujer en Valencia. Social and Education History, 8(1), 23-56. doi: 10.17583/hse.2019.3544

To link this article: http://dx.doi.org/10.17583/hse.2019.3544

\section{PLEASE SCROLL DOWN FOR ARTICLE}

The terms and conditions of use are related to the Open Journal System and to Creative Commons Attribution License (CCAL). 


\section{Music Education at the Institution for the Education of Women in Valencia}

Esperanza Clares-Clares

Universidad de Murcia
Elena Micó-Terol

IES Jaume I Ontinyent (Valencia)

\section{Abstract}

The Institution for the Education of Women (IEM), founded in 1888 in Valencia (Spain), like the Free Institution of Education in Madrid, encouraged musical education of Valencian women. The organization had a network of training schools in Valencia, such as the Elementary School, the School of Secondary Education, the School of Governesses and the School of Fine Arts, which taught subjects like Music Theory, Piano and Singing. The IEM stressed the importance of musical studies, considering them a professional alternative for women. After a brief introduction on education and professionalization of women, in this paper we analyze the role of music in the Institution for the Education of Women of Valencia, the organization, the ideal of education, curriculum development, faculty and other non-educational musical activities developed by the Valencian Institution.

Keywords: Institution for the Education of Women, Music education and women, Institutionalism, Valencia, XIX and XX centuries. 


\section{La Educación Musical en la Institución para la Enseñanza de la Mujer en Valencia}

Esperanza Clares-Clares

Universidad de Murcia
Elena Micó-Terol

IES Jaume I Ontinyent (Valencia)

\section{Resumen}

La Institución para la Enseñanza de la Mujer (IEM), fundada en 1888 en Valencia (España), a semejanza de la Institución Libre de Enseñanza madrileña, fomentó la educación musical de la mujer valenciana. La entidad contó con una red de escuelas de formación, tales como la Escuela Primaria, la Escuela de Segunda Enseñanza, la Escuela de Institutrices y la Escuela de Bellas Artes, en las que se impartían Solfeo, Piano y Canto. La IEM insistió en la importancia de los estudios musicales, considerándolos una alternativa profesional para la mujer. Tras una breve introducción sobre la educación y profesionalización de la mujer, en este trabajo analizamos el papel de la música en la Institución para la Enseñanza de la Mujer de Valencia, la organización, el ideal educativo, los planes de estudios, el profesorado y otras actividades musicales no educativas desarrolladas por la institución valenciana desde su creación hasta su extinción tras la Guerra Civil.

Palabras clave: Institución para la Enseñanza de la Mujer, Educación musical y mujeres, Institucionismo, Valencia, siglos XIX y XX 
I n este trabajo nos proponemos analizar la educación musical que recibió la mujer valenciana a través de la Institución para la Enseñanza de la Mujer (IEM). Esta institución se fundó en 1888 en Valencia, a semejanza de la Institución Libre de Enseñanza de Madrid. La entidad contó con una red de escuelas de formación en Valencia, como la Escuela Primaria, la Escuela de Segunda Enseñanza, la Escuela de Institutrices y la Escuela de Bellas Artes, en las que se impartían materias como Solfeo, Piano y Canto. Desaparecida después de la Guerra Civil (Esteban Mateo, 1974, vol. II, p. 4 señala que la Junta Rectora presidida por la inspectora Ruiz Vallecillo fue la última encargada del edificio y material de la Institución tras la Guerra Civil), la IEM insistió en la importancia de los estudios musicales, considerándolos una alternativa profesional para la mujer.

Tras una breve introducción sobre la educación y profesionalización de la mujer, en este artículo analizaremos el papel de la música en la Institución, la organización, el ideal educativo, los planes de estudios, el profesorado y otras actividades musicales no educativas desarrolladas por la institución valenciana.

Para realizar esta investigación hemos visitado diversos archivos y bibliotecas de Valencia y Madrid, en los que hemos consultado: memorias de curso, programas informativos ("prospectos"), reglamentos, discursos de apertura de curso y otras publicaciones literarias, así como prensa de la época. Es preciso señalar que el corpus documental más importante de la Institución valenciana no se conserva o no se ha localizado hasta la fecha. Las fuentes que existen hoy día son, en relación a la documentación que la citada institución debió generar durante los casi sesenta años de trayectoria, ínfimas, y además están muy diseminadas. La Institución para la Enseñanza de la Mujer de Valencia ha sido objeto de un estudio de Reig Ferrer (2012), que es la más reciente y extensa investigación sobre esta entidad, que viene a sumarse a otros trabajos anteriores, como los de Esteban Mateo (1974), Bueno Ortuño (1979) y Blasco Carrascosa (1980, 1982, 1984). 


\section{Educación Musical y Profesionalización de la Mujer Valenciana en el Siglo XIX}

\section{Hacia la Profesionalización de la Mujer}

En el siglo XIX la mujer española era mayoritariamente analfabeta y tuvo un acceso a la instrucción más limitado que el hombre: en 1900 sólo un $25 \%$ de las mujeres españolas sabían leer y escribir (Canes Garrido, 1990, p. 87). La mujer era educada para ser buena ama de casa, madre y esposa y el estereotipo femenino vigente estuvo íntimamente ligado al rol que ésta debía ejercer en sociedad (Cantizano Márquez, 2004). Era, en términos de la época, el "ángel del hogar" y este papel que la mujer debía cumplir condicionó su instrucción: “¿la compañera del hombre, el ángel del hogar que es para lo que Dios la ha criado, no merece que cultive su inteligencia para hacer más dulce y llevadera la vida de los que la rodean, sean éstos padres, hermanos o esposos?" (Alarcón, 1890, p. 10).

En líneas generales, aquellas mujeres que podían recibir una educación reglada solían pertenecer a la aristocracia y clase media. En las escuelas femeninas se enseñaba lectura, escritura, costura y bordado, moral y religión y en los casos de una formación más completa se impartían nociones básicas de economía, geografía, historia, francés y "asignaturas de adorno", es decir, música y dibujo (Sánchez de Andrés, 2008, p. 56). La profesora de la IEM, Julia Alarcón, lo describía así:

¿Qué se hace con la mujer por regla general?

Se la enseña a leer; en la mayor parte de los casos muy medianamente a escribir, alguna que otra labor de utilidad problemática, y como nota sobresaliente algo de música o pintura, aprendido por puro pasatiempo.

Ya lo veis; con tan menguada instrucción, no os debe extrañar que la mujer no piense con seriedad de ciertas cosas; que en sus conversaciones se muestre nimia y pueril, y por consiguiente que preste poca atención a todas aquellas que inspiran algún interés. 
[...] Preciso es que seamos consecuentes; si la mujer ha nacido para ser la compañera del hombre, es necesario que no sea extraña a sus conocimientos (Alarcón, 1890, p. 10).

Desde el punto de vista legislativo, la primera mitad del siglo XIX se caracterizó por la indefinición en torno a la educación femenina (Sole Romero, 1990, pp. 33-44; Palacio, 1992, pp. 110-121¹). La Ley Moyano de 9 de septiembre de 1857 supuso una significativa primera mejora legal porque contempló la creación de escuelas tanto para niños como para niñas en pueblos de más de quinientos habitantes, y en los de menor número, una escuela mixta con diferenciación de sexos. La nueva ley proponía la creación de Escuelas Normales femeninas y reconocía también, por primera vez, la conveniencia de dar una formación pedagógica a las maestras. No obstante, esta ley de instrucción pública no preparaba a la mujer para ningún trabajo que no fuera el hogar o el magisterio (véase Figura 1).

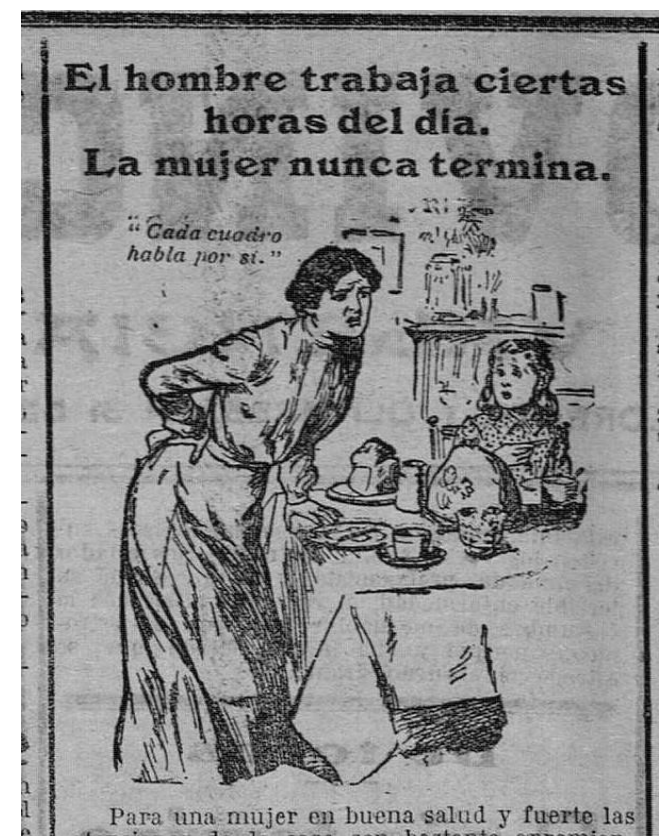


Figura 1. Viñeta "El hombre trabaja ciertas horas del día. La mujer nunca termina". Fuente: Las Provincias, 10-12-1915.

Durante el Sexenio revolucionario, los krausistas emprendieron una serie de medidas eficaces encaminadas a mejorar la educación de la mujer española, igualando sus derechos a los del hombre, pero sin contravenir los roles de esposa y madre. El rector de la Universidad Central y antiguo franciscano Fernando de Castro (1814-1874), en su empeño por fomentar la educación de la mujer española, promovió la creación del Ateneo Artístico y Literario para Señoras en Madrid (febrero de 1869) y paralelamente organizó unas Conferencias Dominicales para la educación de las mujeres (febrero a mayo de 1869). Aunque en ellas se trataron diversos temas, todas reivindicaron iguales derechos y deberes en materia de instrucción para hombres y mujeres, según las misiones que unos y otros tenían asignadas. La mujer, según los krausistas, debía estar capacitada para "compartir las preocupaciones de un esposo progresista y saber comunicar esas ideas a sus hijos" (Alcázar Cruz Rodríguez, 2007, pp. 17-18). También por iniciativa de Castro nació el 1 de diciembre de 1869 la Escuela de Institutrices de Madrid y en octubre de 1870, la Asociación para la Enseñanza de la Mujer (Alcázar Cruz Rodríguez, 2007; Mandado Gutiérrez et al., 2011; Ontañón Sánchez, 2003; Parreño Arenas, 2011; Sánchez de Andrés, 2009b; Sole Romero, 1990; Vázquez Ramil, 2001). La Asociación madrileña contó además con una Sección de Idiomas y Música (1878), la Escuela de Comercio (1878), la Escuela de Correos y Telégrafos (1883) y una Escuela Primaria (1884). En Valencia, el fenómeno krausista dio lugar a la creación de la IEM en 1888, como veremos más adelante.

Durante la segunda mitad del siglo XIX, otras corrientes pedagógicas de carácter laico fomentaron la educación de la mujer como vehículo indispensable para lograr su emancipación. En general, estas iniciativas innovadoras tuvieron un carácter anticlerical, fueron afines a la ideología librepensadora y se consolidaron en las últimas décadas de la centuria decimonónica. El movimiento racionalista valenciano, por ejemplo, se caracterizó por la implicación de un colectivo de librepensadores y republicanos que, sobre prácticas previas, quiso consolidar un modelo laico y alternativo desde finales del siglo XIX y sobre todo a partir de 1901 con la 
creación de escuelas laicas (Lázaro, 1992). También en Valencia, en 1896, un grupo de mujeres librepensadoras comprometidas con la igualdad de derechos y deberes entre hombres y mujeres crearon la revista semanal $L a$ Conciencia Libre, y un año más tarde fundaron la Asociación General Femenina (Sanfeliu, 2005, pp. 95-96). Los objetivos primordiales de esta Asociación fueron la instrucción y educación de la mujer y el laicismo en la enseñanza. Por iniciativa de la Asociación General Femenina se fundaron escuelas nocturnas y diurnas para niñas y mujeres y gabinetes de lectura, entre otros (Sanfeliu, 2005, pp. 108-111).

La proliferación de escuelas basadas en estas pedagogías innovadoras fue un fenómeno común a otras ciudades españolas. Véanse los trabajos de Marín Silvestre (2018) y Palà Moncusí (2018) quienes proponen un análisis de la influencia del espiritismo en la sociedad barcelonesa de principios del siglo XX y el librepensamiento catalán, respectivamente. El ideario modernizador y laico de estas nuevas corrientes favoreció la participación de la mujer en espacios públicos que tradicionalmente le eran vetados y desde los que, posteriormente, reivindicaron un rol modernizado de identidad femenina alejado del discurso doméstico (Palà Moncusí, 2018, p. 260).

\section{Educación Musical y Mujeres en Colegios, Academias y Sociedades Valencianas}

La música no estaba contemplada en los planes de estudios de la Primera y Segunda Enseñanza y solo se ofertó en algunos centros, casi siempre de carácter privado. En esas ocasiones, la música se incluyó en las materias de ampliación, complementarias o "de adorno". Para cursarlas, a menudo las familias interesadas debían pagarla aparte. En Valencia, hubo colegios que ofrecían clases de música, como la Casa Enseñanza, dirigida por Catalina Lucena, el Colegio Valentino, dirigido por Isabel Coll, el Colegio Edetano, por María Gomis, y el Colegio Sagrado Corazón, entre otros (Pinedo Herrero, Más Zurita, y Mocholí Roselló, 2003, p. 45; Sela, 1889b, p. 353)². De este modo, la música se convertía en una materia elitista, reducida a una minoritaria clase media y alta y, por ende, de imposible acceso a los menos favorecidos socialmente. Esta precaria situación afectó especialmente a los colegios públicos, escasos en número y frecuentemente mal acondicionados por la falta de inversión. Ello acentuó las desigualdades sociales y 
económicas que, en el ámbito de la enseñanza, entrañaron diferentes oportunidades para las clases altas en detrimento de las bajas.

Durante la segunda mitad del siglo XIX se incrementó el número de escuelas, entre otras circunstancias que mejoraron el panorama, aunque la música siguió ausente de la oferta educativa reglada hasta el final del siglo. En 1884, se incluyó la enseñanza del "Canto" en los planes de estudios de las escuelas de párvulos. Posteriormente, en 1901, la enseñanza de esta materia se introdujo en todas las escuelas y grados de la enseñanza primaria (Clares-Clares, 2017, pp. 56-57).

En los estudios de magisterio de la Escuela Normal Central de Maestros y Maestras se incorporó la asignatura "Música y Canto" en 1878. Sin embargo, en las Escuelas Normales de provincias esta incorporación no se produjo hasta 1898 y, además, limitada exclusivamente a los dos cursos del grado superior. Con esta planificación se mantuvo en los planes de 1900, 1901 y 1903, por lo que los maestros de enseñanzas elementales (que cursaban únicamente los dos primeros cursos) no recibían oficialmente preparación musical (Clares-Clares, 2017, pp. 57-58) (véase Figura 2).

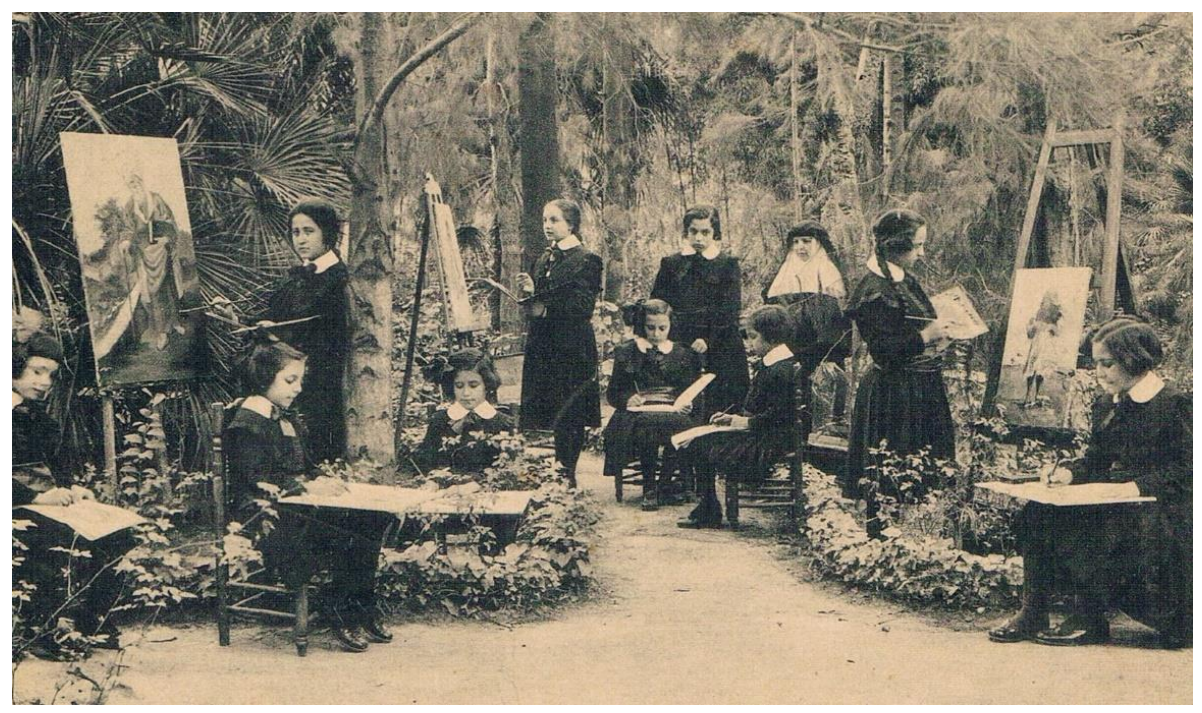


Figura 2. Alumnas recibiendo una clase de Dibujo en el Colegio Santa Ana de Valencia (1913). Fuente: Archivo Particular de Rafael Solaz Albert.

Las sociedades valencianas también ofertaron música (entre otras materias como escritura, primeras letras y dibujo) a todas las capas sociales, incluida la mujer (Micó Terol, 2014, pp. 79-101). Entre ellas destacó particularmente la Sociedad Económica de Amigos del País de Valencia, que inauguró en 1851 una Escuela Popular de Música Vocal dirigida por Pascual Pérez Gascón. La contribución de la Real Sociedad Económica de Amigos del País de Valencia fue decisiva para fomentar el interés por la música en esta ciudad, y gracias a su iniciativa nació en 1879 el Conservatorio de Música de Valencia (Fontestad, 2011), cuyas enseñanzas también iban dirigidas a mujeres. La Diputación Provincial y el Ayuntamiento de Valencia contribuyeron con subvenciones significativas al desarrollo de este proyecto, al igual que hizo con la Escuela de Comercio valenciana y la Institución para la Enseñanza de la Mujer, de las que más tarde nos ocuparemos. Fuera del ámbito musical, la Real Sociedad Económica de Amigos del País de Valencia prestó especial atención a la educación general de la mujer. En 1881, por ejemplo, impulsó la creación de una Asociación de Señoras para el establecimiento de escuelas dominicales de adultas (Palacio, 1992, pp. 132-133).

Según Fontestad (2011, pp. 21, 45-52), las primeras escuelas municipales de música de Valencia surgieron a petición de varios profesores de música, que se ofrecieron al Ayuntamiento de la ciudad para colaborar gratuitamente. En 1867 y 1868, el Ayuntamiento valenciano concedió licencias a Manuel Penella Raga y Consuelo del Rey, respectivamente, para fundar las primeras escuelas municipales de música, y en los años siguientes, la corporación municipal autorizó la creación de nuevos centros de enseñanza.

En Valencia, también hubo diversas academias de música privadas, algunas de las cuales pertenecían a entidades culturales que sólo permitían el acceso de sus socios, como la Academia Filarmónica del Liceo Valenciano. Todas las entidades pedagógicas mencionadas, además de ocuparse del aspecto didáctico, organizaban conciertos y colaboraban con otras entidades corales y teatros. 


\section{La Institución para la Enseñanza de la Mujer de Valencia}

\section{El Institucionismo en Valencia}

El Krausismo español y su posterior desarrollo institucionista fue una corriente ideológica de gran influencia en la vida cultural y artística española (Tahull, Montero y Molina, 2016). Basado en las teorías del filósofo alemán Friedrich Krause (1781-1832), el krausismo surgió por oposición al pensamiento intelectual tradicional del régimen conservador de la Restauración. Fue introducido por Julián Sanz del Río, entre otros intelectuales, y tuvo vigencia en España entre 1854 y 1936. Los krausistas cultivaron especialmente los temas de ética, derecho, sociología y pedagogía, y promovieron un vasto movimiento de educación popular que dio como resultado la creación en 1876 de la Institución Libre de Enseñanza de Madrid (ILE). La creación de la ILE se enmarcó en el espíritu regeneracionista del cambio de siglo, que consideraba la educación en lugar destacado y planteaba una profunda renovación pedagógica, en la que la música adquiría una nueva valoración. El Krausismo español y el Institucionismo prestaron especial atención a la educación de la mujer.

A través de Eduardo Pérez Pujol (1830-1894), José Villó y Ruiz (18391907), Eduardo Soler (1845-1907) y Alfredo Calderón Arana (1850-1907), interesados en el Krausismo desde su época de estudiantes universitarios, se introdujo en Valencia esta filosofía a mitad del siglo XIX. Algunos de sus continuadores fueron: Saturnino Milego e Iglada (1850-1929), Aniceto Sela (1863-1934), Luis Morote y Creus (1862-1913), Rafael Altamira y Crevea (1866-1951), José Deleito y Piñuela (1879-1957); y discípulos de Giner y Cossío, como Pascual Carrión y Carrión (1891-1976), José Navarro Alcácer (1891-1987) y Angelina Carnicer Pascual (1893-1980) (Delgado Criado, 1994, p. 457; Esteban Mateo, 1974, pp. 1-24). Bajo su influencia se promovió la creación de diversos organismos socio-educativos en la ciudad, como la Escuela de Comercio para Señoras (11 de julio de 1883); la Institución para la Enseñanza de la mujer (1888-89); la Extensión Universitaria (inaugurada en el curso 1902-03); la Universidad Popular (creada por Blasco Ibáñez el 8 de febrero de 1903); el Instituto-Escuela (creado por Decreto de 2 de marzo de 1932); la Escuela Cossío (octubre de 1930); las Misiones Pedagógicas (Decreto de 29 de mayo de 1931) y la Junta 
para la Ampliación de Estudios (diciembre de 1936) (Delgado Criado, 1994, pp. 461-465; Esteban Mateo, 1974, vol. II, pp. 3-24).

\section{La Escuela de Comercio para Señoras (1883)}

El origen de la IEM valenciana se remonta a abril de 1881, momento en el que la Sociedad Económica de Amigos del País de Valencia constituyó una comisión encargada de estudiar la posibilidad de crear una Escuela de Institutrices y una Escuela de Comercio para Señoras en Valencia. La comisión debía encargarse de resolver la financiación y buscar profesorado y local. Sin embargo, al no contar con suficientes recursos, se desestimó la creación de la Escuela de Institutrices para centrarse en el proyecto de la Escuela de Comercio para Señoras, fundada finalmente el 11 de julio de 1883. Juan Antonio Oliver Andrés, socio de la Económica, fue uno de sus principales promotores. La Escuela inauguró sus clases el 23 de noviembre de 1884 con ocho alumnas en el local de la Escuela Normal de Maestras de Valencia. Las fuentes documentales que recogen la fundación de la Escuela de Comercio y la posterior Institución para la Enseñanza de la Mujer de Valencia aparecen citadas en el apartado "Fuentes" al final de este trabajo.

El objetivo de la Escuela de Comercio, según su Reglamento, era ofrecer a las mujeres instrucción propia para el ejercicio de la profesión mercantil. Estos estudios otorgaban el Título de Aptitud Mercantil y sus materias se organizaban en dos cursos en los que no se incluía música (Escuela de Comercio, 1884, pp. 3-4).

La favorable acogida del Título de Aptitud Mercantil permitió la ampliación paulatina de otras enseñanzas: un Curso Especial Preparatorio y los Estudios de Ampliación (véase Tabla 1). Estos últimos se impartieron al menos desde el curso 1887-88, aunque no hemos hallado documentación de los dos cursos anteriores. El Curso Especial Preparatorio tenía como finalidad 1) ampliar la instrucción primaria de las alumnas y 2) prepararlas para estudios posteriores (IEM, 1893, pp. 1-2). Los Estudios de Ampliación posibilitaban completar conocimientos en diferentes materias, como idiomas, dibujo y música (Sela, 1888a, pp. 109-111). 


\section{Clares-Clares \& Micó-Terol-La educación musical}

Tabla 1

Estudios ofertados por la Escuela de Comercio para señoras de Valencia, durante el curso 1887-1888. (Elaboración propia a partir de Sela, 1888a, p. 109)

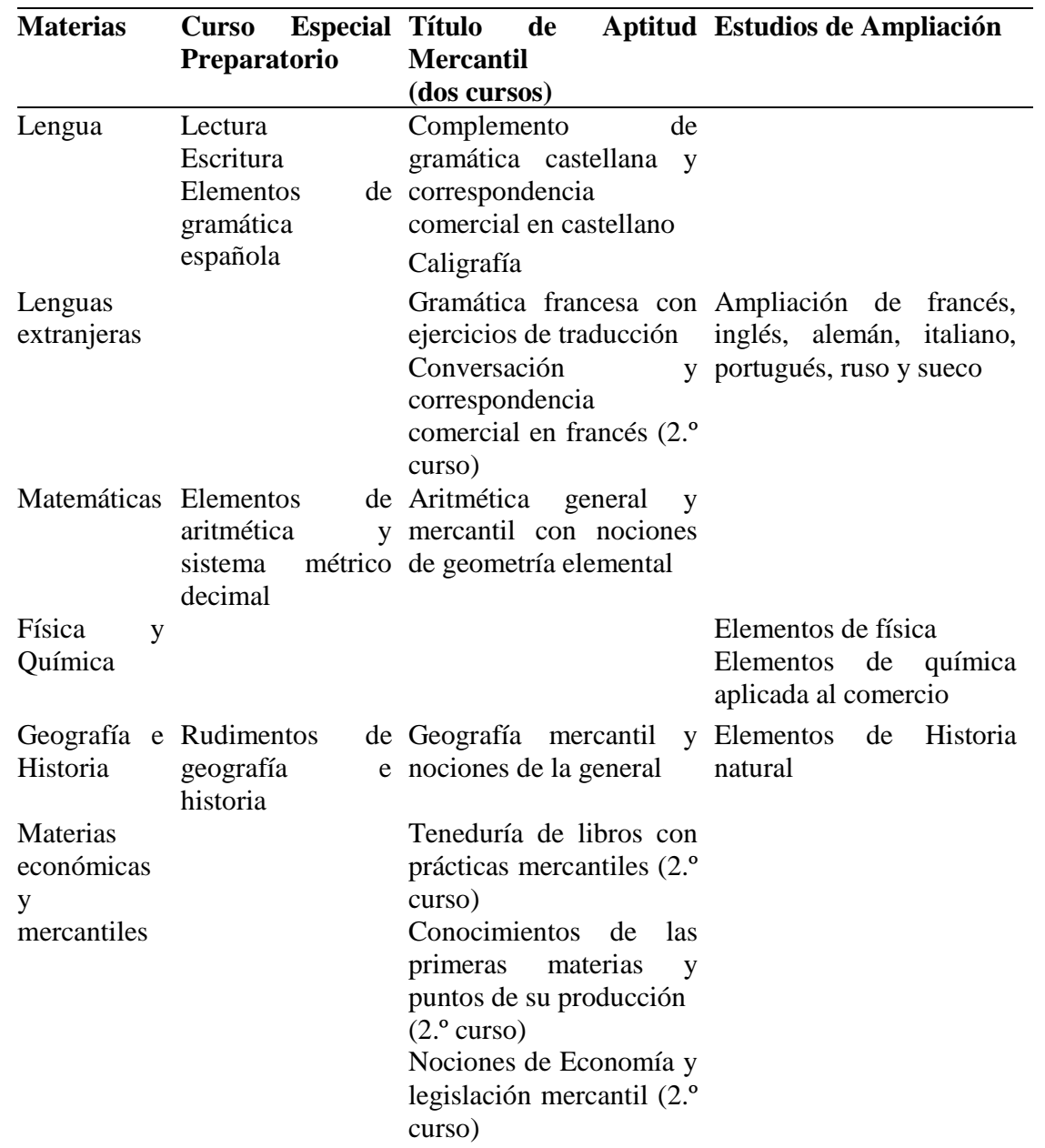


Tabla 1

Estudios ofertados por la Escuela de Comercio para señoras de Valencia, durante el curso 1887-1888. (Elaboración propia a partir de Sela, 1888a, p. 109) (continuación).

$\begin{array}{llr}\begin{array}{l}\text { Hogar } \\ \text { vida diaria }\end{array} & \begin{array}{l}\text { Elementos de } \\ \text { higiene doméstica }\end{array} \\ & \begin{array}{l}\text { Nociones de } \\ \text { derecho de uso } \\ \text { frecuente } \\ \text { general aplicación } \\ \text { a la vida }\end{array} \\ \text { Religión } & \begin{array}{l}\text { Principios de } \\ \text { religión y moral } \\ \text { Dibujo } \\ \text { Elementos de } \\ \text { dibujo }\end{array} \\ \text { Música } & \text { Nociones } \\ & \text { música de }\end{array}$

Dibujo de figura con nociones de colorido y pintura a la acuarela Solfeo, piano y canto

\section{La Creación de la Institución para la Enseñanza de la Mujer (1888) y su Trayectoria Posterior}

En 1888, la Escuela de Comercio cambió de denominación a Institución para la Enseñanza de la Mujer (así consta en el expediente C. 258, III-Educación $\mathrm{n}^{\mathrm{o}} 1$, "Documentación relativa a la constitución de la Institución de la Enseñanza de la Mujer y su Reglamento (1892)" del Archivo de la Sociedad Económica de Amigos del País de Valencia). Con este último nombre se mantuvo durante todo el periodo que hemos estudiado.

La Institución, que fue reglamentada oficialmente en diciembre de 1888, estaba presidida por una Junta Directiva, compuesta por los profesores numerarios de la IEM además de la Directora de la Escuela Normal de Maestras de Valencia. Los asuntos económicos recaían en la Junta General, formada por un presidente y dos vicepresidentes, entre otros cargos, además de los representantes de las corporaciones patrocinadoras (Real Sociedad Económica de Amigos del País, Ayuntamiento y Diputación de Valencia) y socios colaboradores. Los socios se dividían en tres categorías, en función de las cuotas que éstos aportaban a la IEM (1890, pp. 10-11). 
Desde su fundación en 1888, la IEM amplió las opciones de formación para mujeres con dos tipos de estudios: aquellos que dotaban a la mujer de una educación básica y general, es decir, no profesional y los estudios profesionales, destinados a formar a las alumnas para la obtención de un futuro empleo.

En el curso académico 1888-1889, se incorporaron los estudios de Cultura General (o de Segunda Enseñanza). Para ingresar en estos estudios, las alumnas debían tener superadas las materias correspondientes a Primaria y tener más de diez años. La finalidad de los estudios de Cultura General o Segunda Enseñanza era:

proporcionar a la mujer los conocimientos que hoy resultan indispensables para vivir en una sociedad culta y para contribuir a la educación de las futuras generaciones. Teniendo en cuenta la escasa preparación con que las alumnas de instrucción primaria salen de las escuelas oficiales, se ha mantenido también el curso preparatorio que tan excelentes resultados ha producido en los años anteriores (IEM, 1889, p. 1).

Durante este curso también quedaron definitivamente formadas las Escuelas de Idiomas y Bellas Artes (esta última dividida en Sección de Dibujo y Sección de Música), que procedían de los Estudios de Ampliación.

En el curso 1889-1890 se añadió la Escuela de Institutrices (Sela, 1889b, p. 6), cuyos requisitos de acceso eran tener cumplidos doce años y superar un examen de contenidos generales, entre ellos, "Principios de Solfeo". La Escuela de Institutrices tenía un doble propósito:

Puede considerarse a la vez como general y profesional, y de aquí su doble utilidad, pues no sólo proporciona un medio decoroso de vivir, que hoy no aprovechan más que las señoritas extranjeras, sino que es de suma conveniencia para la vida de familia y para el progreso de la educación nacional el que las madres puedan guiar conscientemente los primeros pasos de sus hijos (IEM, 1891, p. 2).

En el curso académico 1896-1897 ya aparece la Escuela Profesional en la documentación consultada, por lo que debió implantarse en alguno de los dos cursos anteriores. En esta escuela se estudiaba asignaturas como 
"Labores de adorno y corte" y "Corte y confección de trajes y sombreros de señoras" (IEM, 1896, p. 2).

En el curso 1898-1899 se añadió la Escuela Primaria (a la que se accedía con cinco años cumplidos) que constituía "el comienzo de la obra educativa, la que tiene la misión de guiar a la mujer en su más temprana edad y prepararla para adquirir con fruto los conocimientos que necesita" (IEM, 1899, p. 1).

En los años siguientes, el proyecto educativo de la IEM impulsó la creación de nuevos centros. El 2 de noviembre de 1905 se inauguró la Escuela Nocturna de Adultas cuyo fin era "favorecer la cultura de la clase artesana" y antes de 1912, la IEM incorporó una Escuela del Hogar, una Escuela de Lencería y Bordado, así como cursos especiales de "Labores de corte y confección" y "de escritura a máquina" que sustituían a los antiguos estudios de la Escuela Profesional (Oliver, 1905, p. 2). En 1933, poco antes de su desaparición, la Institución contaba con las siguientes escuelas: Escuela de Primaria Graduada, Escuela de Segunda Enseñanza, Escuela de Comercio, Escuela de Institutrices, Escuela del Hogar, Escuela de Idiomas, Escuela de Perfeccionamiento, Escuela de Bellas Artes, Escuela de Lencería y Bordados, Escuela de Preparación Especial para el Instituto, Escuela de Comercio (La Correspondencia de Valencia, 1933, p. 2).

Al margen de la actividad educativa de la IEM, canalizada a través de sus diferentes escuelas, la institución promovió otras iniciativas siempre con la pretensión de elevar la condición social y cultural de la mujer.

En 1915 se creó la Asociación de Profesoras y Alumnas de la IEM para 1) procurar el ahorro, el auxilio y el seguro mutuo de sus asociadas; 2) cooperar con la Institución y 3) trabajar para la mejora de "la situación social, intelectual, moral, jurídica y económica de la mujer” (IEM, 1915, p. 4). La Asociación se estructuró en seis secciones diferentes, cuyos cometidos eran:

- Beneficencia: ayudar y socorrer a los necesitados.

- Enseñanza: gestionar el establecimiento de escuelas de cocineras, de planchadoras, de niñeras y de cuidados y asistencia a enfermos, entre otras, en la IEM. Además, debía velar por la "desmoralización de la juventud y cooperar a la obra pacifista de la mujer" (IEM, 1915, p. 13).

- Biblioteca Circulante: difundir la cultura en la mujer española. 
- Higiene: hacer desaparecer el alcoholismo y la tuberculosis, procurar protección y asistencia a la mujer pobre y embarazada, establecer colonias escolares y difundir la limpieza, el aseo, el esmero y la pulcritud en todos los actos y manifestaciones de la vida.

- Artes y Excursiones: organizar conferencias artísticas o literarias, visitas a museos y otros monumentos artísticos, así como audiciones musicales.

- Reforma Social: procurar el acceso de la mujer a todos los empleos y estudios para los que se considerase apta, velar para que sus trabajos obtuvieran la remuneración justa y sin diferencia de sexos, elevar la condición social de la mujer, procurar leyes que favorecieran la igualdad en el Código Civil y educar a la mujer en su derecho a elegir y ser elegida.

En 1923 la IEM inauguró una biblioteca, con fondos obtenidos casi mayoritariamente por donación (Las Provincias, 1923, p. 4), y organizó conferencias dominicales regulares. Las conferencias iban dedicadas principalmente a mujeres jóvenes, pero estaban abiertas al público en general y tuvieron una magnífica acogida (Las Provincias,1903b, p. 2). Las conferencias eran variadas y abarcaban, entre otras, materias de Historia Universal, Lengua, Literatura y Educación. Algunos ponentes y títulos fueron: Manuel Candela, "Prejuicios e injusticias sociales en el concepto educativo de la mujer"; María Carbonell, "La tradición y el ideal en lo que se refiere a la educación femenina" y Carmen Cervera, "La naturaleza y destino de la mujer" (Las Provincias, 1903a, p. 2; Las Provincias, 1903c, p. 2).

Desde 1884 hasta 1888, la IEM compartía edificio con la Escuela Normal de Maestras, aunque un año después la sede se trasladó al segundo piso del antiguo palacio de los condes de Parcent, situado en la calle Juan de Vilarrasa, 12. En el nuevo local se habilitaron seis aulas para las distintas escuelas, con mobiliario actualizado según los modelos recomendados por el Museo Pedagógico de Instrucción Primaria de Madrid y dependencias destinadas a Biblioteca, Secretaría y Sala de Gimnasia, entre otras. Desconocemos si existía una sala específica para las clases de música. Nos consta, en cambio, que la Institución compró un piano Gómez para la 
sección de música en el curso 1888-1889, gracias a la subvención de la Real Sociedad Económica de Amigos del País de Valencia (Sela, 1889b, p. 10).

A partir de 1917, la IEM ocupó una casa en planta baja que estuvo ubicada en el Paseo de la Alameda, 29 de Valencia (La Correspondencia de Valencia, 1917, p. 2). Véase Figura 3. El nuevo local "cerrado con verja y rodeado de un frondoso jardín que constituye un higiénico y bello campo de esparcimiento y de recreo para la población escolar" (La Correspondencia de España,1925, p. 2). De hecho, "las clases se dan al aire libre, rodeadas de hermosos jardines que favorecen su organismo, cautivan el espíritu y elevan el gusto y la inteligencia" (La Correspondencia de Valencia, 1933, p. 2).

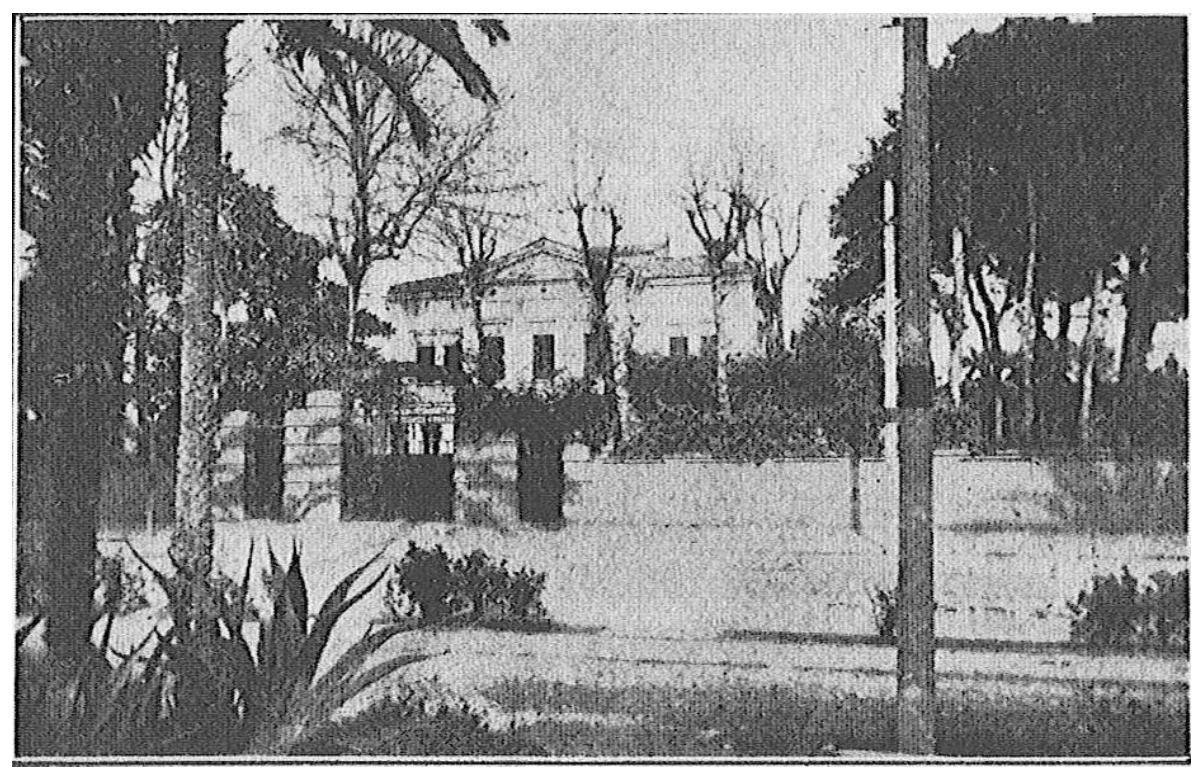

Figura 3. Sede (actualmente desaparecida) de la Institución para la Enseñanza de la Mujer en el Paseo de la Alameda, no 29 de Valencia en 1923 (Oliver, 1923, p. 2).

\section{Ideal Educativo de la IEM}

Los institucionistas valencianos buscaban la regeneración social a través de la enseñanza. Su objetivo era "educar" (no sólo enseñar o instruir) a hombres 
y mujeres "capaces de dirigirse en la vida y de ocupar digna y útilmente el puesto que les está reservado" (Delgado Criado, 1994, p. 460). Sin embargo: "¿Tiene la mujer aptitudes para recibir una instrucción amplia? Si las tiene, ¿es conveniente que la reciba para el cumplimiento de los fines que ha de realizar en el orden moral y material?" (Alarcón, 1890, p. 4) 3 .

Estas preguntas, que fueron formuladas por la profesora de la IEM Julia Alarcón, y de controvertidas respuestas para la época, resumen los debates contemporáneos en torno a la educación de la mujer.

Para el abogado valenciano y fundador de la IEM, Juan Antonio Oliver, se debía garantizar y facilitar la instrucción a "todo ser racional" para conseguir que cada individuo (sin distinción de sexos) desarrollara su propia naturaleza y destino individual; negar este derecho a la mujer sería frenar el progreso de la Humanidad. Sin embargo, los "seres racionales, si bien son todos iguales entre sí por su naturaleza, son, sin embargo, esencialmente desiguales como individuos" (Oliver, 1887, p. 11). Para el autor, una de las principales desigualdades es el género, puesto que hombres y mujeres "tienen facultades diversas y destinos diferentes que realizar en la vida" (Oliver, 1887, p. 11). En el hombre, según Oliver, predomina "la fuerza, el vigor, la energía," y en la mujer "la flexibilidad, gracia, elegancia y belleza". En lo intelectual, "la mujer posee una intuición más rápida, una imaginación más viva y precoz que la facilita adquirir cierto grado de cultura más pronto que el hombre" (Oliver, 1887, p. 12). Según este pensamiento, los sexos se oponían pero no se excluían ni se subordinaban. La mujer era, ante todo, madre de familia y, por tanto, transmisora de valores a sus hijos. Su falta de instrucción privaría a la madre de cumplir sus obligaciones para con sus hijos; de ahí la obligación de educarla. En resumen, cada familia debía convertirse en una verdadera escuela: educar a la mujer era educar a la sociedad.

A diferencia de otros intelectuales del momento, Oliver defiende el acceso de la mujer a las enseñanzas superiores, a pesar de que hombres y mujeres tienen para él aptitudes distintas: mientras que la mujer podía ejercer el magisterio, el comercio o incluso la medicina o farmacia, consideraba que "no deben ocupar cargos políticos, ni los empleos de la magistratura, ni los grados de la milicia, ni la mayor parte de las funciones civiles" (Oliver, 1887, p. 14). 
En líneas generales, los principios básicos por los que abogaba la IEM eran: "la coeducación, la enseñanza individual, la enseñanza cíclica, sustitución de los libros de texto por cuadernos de notas breves, excursiones y sobre todo, libertad de cátedra, autonomía y laicismo escolar" (Oliver, 1887, p. 14).

Este ideario seguía las pautas de la Institución Libre de Enseñanza de Madrid, que reconocía el derecho de la mujer para desarrollar todas sus facultades de la manera más completa posible: educación por encima de la mera instrucción para formar su carácter, desarrollar su propio pensamiento y fortalecer su autonomía individual para enseñarla a vivir. Los criterios pedagógicos se asentaban sobre las bases de una enseñanza intuitiva, práctica, basada en el trabajo experimental y no memorístico. El Prospecto del curso académico 1891-1892 lo explicaba claramente:

[...] por el método intuitivo, haciendo ver las cosas antes que las palabras, y sustituyendo a la repetición mecánica de las ideas contenidos en un libro, el trabajo personal de la alumna, en el sentido de desarrollar su pensamiento y examinar en su propia conciencia las ideas pero con el auxilio, pero nada más que con el auxilio, del profesor. En dos palabras: enseñanza realista, en vez de la verbalista y libresca (IEM, 1891, p. 2).

El claustro de profesores durante el periodo de años estudiado es extenso y contó con importantes institucionistas como Aniceto Sela, quien abandonó la Institución por su traslado a Oviedo en 1891; Eduardo Soler fue miembro de la Junta General de la IEM desde 1894, así como bibliotecario y director de excursiones, entre otros cargos; José Deleito fue profesor de la Institución entre 1907-14 y Juan Antonio Oliver, como apuntábamos anteriormente, creador de la misma. Los prospectos que anualmente imprimía la IEM, así como las memorias de curso y la prensa local, ofrecen listados completos del profesorado de la Institución durante el periodo estudiado. Véase el apartado "Fuentes" al final de este trabajo. 


\section{La Música en la IEM}

La música era privilegiada dentro del ideal educativo de la IEM. No sólo estuvo presente en la mayoría de las titulaciones ofertadas por ella sino que llegó a formar una sección propia que incluía especialidades de Solfeo (en diferentes niveles), canto y piano. La importancia que se le dio a la música en la institución valenciana coincide plenamente con los ideales educativos en torno a la música de su homónima madrileña. Para los institucionistas, la música debía formar parte de la educación integral del hombre. En otras palabras, debía formar parte de la base de la cultura general a la que todos los ciudadanos debían acceder (Sánchez de Andrés, 2009b, pp. 252-262).

\section{La Música en los Planes de Estudios de la IEM}

La música se enseñaba en la IEM con un propósito claro. En la Memoria del curso 1889-1890, Aniceto Sela afirmaba:

No ha de tratarse en nuestra Escuela de formar artistas (misión que llenan cumplidamente el Conservatorio de Música y la Academia de Bellas Artes), sino de desarrollar y cultivar el sentimiento y el gusto de las alumnas y de completar su educación con la educación estética. Pero tampoco han de considerarse estas enseñanzas cual objeto de adorno, como aún se las denomina con notorio desconocimiento de sus verdaderos fines (Sela, 1889b, p. 5).

En la institución valenciana la música se concretó en varias asignaturas denominadas "Solfeo", "Canto" y "Piano", además de otra genéricamente llamada "Música".

La iniciación de la música tenía lugar en el Curso Especial Preparatorio (después llamada Escuela Preparatoria), en la que se ofrecía nociones elementales de Solfeo. Los Estudios de Ampliación, que sólo tuvieron cabida en los primeros años de la IEM, estaban destinados a completar la formación de las alumnas. Dado que eran estudios complementarios, y a un nivel avanzado, las asignaturas de música debieron ofrecer un mayor contenido conceptual. La música estaba programada en tres cursos de 
Solfeo, otros tres de Piano y otra asignatura de Canto, cuya duración no consta (Sela, 1888a, p. 109; IEM, 1895, p. 280).

En la Escuela Primaria se impartía la asignatura de "Música". Aunque no hemos hallado la planificación, cabe suponer que ésta también se distribuyera en varios cursos, en los que se alternaran el Canto y el Solfeo.

En la Escuela de Cultura General o Segunda Enseñanza y en la Escuela de Institutrices se impartían tres cursos de Solfeo. Al menos desde 1911, otra asignatura de Piano completaba el Solfeo en la Escuela de Institutrices.

En la Escuela de Bellas Artes se impartía Solfeo (tres cursos), Piano (tres cursos) y Canto (no nos consta el número de cursos) (Oliver, 1913, pp. 8081; IEM, 1895, pp. 280-282). La Escuela de Bellas Artes fue una de las más demandadas por las alumnas. La Tabla 2 muestra una relación de alumnas matriculadas en las secciones de Dibujo y Música durante el curso 18891890.

Tabla 2

Número de alumnas matriculadas en la Escuela de Bellas Artes durante el curso 1889-1890 (Sela, 1890, p. 7).

\begin{tabular}{llll}
\hline Sección & Asignatura & Curso & $\mathrm{N}^{\mathrm{o}}$ alumnas \\
\hline \multirow{4}{*}{ DIBUJO } & Dibujo de adorno & $1 .^{\circ}$ & 8 \\
& Dibujo de adorno & $2 .^{\mathrm{o}}$ & 1 \\
& Dibujo de figura & $1 .^{\mathrm{o}}$ & 4 \\
& Dibujo de paisaje & - & 2 \\
& Dibujo del antiguo & - & 1 \\
\hline Total & & & 16 \\
\hline \multirow{5}{*}{ MÚSICA } & Solfeo & $1 .^{\circ}$ & 4 \\
& Solfeo & $2 .^{\circ}$ & 8 \\
& Solfeo & $3 .^{\circ}$ & 1 \\
& Piano & $1 .^{\circ}$ & 12 \\
& Piano & $2 .^{\circ}$ & 4 \\
Total & Piano & $3 .^{\circ}$ & 3 \\
\hline
\end{tabular}




\title{
44 Clares-Clares \& Micó-Terol-La educación musical
}

Tal y como se deduce de esta Tabla 2, las asignaturas de música tenían el doble de matrículas. El éxito de la música aún se hace más notable cuando comparamos estos números con las matriculadas en otras escuelas. Durante ese mismo año, la Escuela Preparatoria contó con 22 alumnas; 7 la Escuela de Comercio; 6 la de Idiomas, frente a las 48 de la Escuela de Bellas Artes (16 y 32 de dibujo y música, respectivamente). Durante el curso siguiente (1890-1891) el número de alumnas matriculadas en la Sección de Música todavía seguía siendo superior a las matriculadas en la de Dibujo (29 frente a 24, respectivamente) (Sela, 1891, p. 7).

La Sección de Música de la Escuela de Bellas Artes tuvo un carácter profesional, aunque con matices. Sus fines quedaron expresados en los siguientes términos:

\begin{abstract}
Rectificada la errónea opinión que consideraba como de mero adorno la cultura artística, no hay necesidad de insistir sobre la importancia que estos estudios ofrecen ya como base de una honrosa profesión, ya como medio de educar y elevar el gusto y el sentimiento (IEM, 1891, p. 3).
\end{abstract}

Subrayamos la expresión "como base de una honrosa profesión" porque entendemos que estos estudios eran insuficientes para la plena dedicación profesional musical de la mujer.

\section{Profesorado y Métodos de Enseñanza Musical}

Durante los años estudiados, los profesores y profesoras que impartieron las clases de música en la IEM valenciana fueron: Amancio Amorós, Adelaida Alarcón, Asunción Peset y Elisa López Roncal.

El pianista y compositor Amancio Amorós (1854-1925) fue el primer profesor de Piano y Solfeo de la IEM, cargo que ejerció entre 1888 y 1901. En 1902 y hasta 1925, Amorós fue profesor de Solfeo y Armonía del Conservatorio de música de Valencia (Micó Terol, 2014, pp. 173, 188 y 190). Adelaida Alarcón fue profesora de Piano en la IEM desde 1888 hasta al menos 1894; y nos consta que también trabajó como profesora de piano en el Conservatorio de Valencia (Fontestad, 2011, p. 366). Asunción Peset fue profesora de Canto en la IEM al menos hasta 1900 (IEM, 1899, p. 3) y la 
pianista Elisa López Roncal fue docente desde al menos 1912 hasta 1935 (Reig, 2012, pp. 133-135).

La documentación consultada permite reconstruir, al menos en parte, la metodología que se empleó en las clases de música. Según el horario del curso 1890-1891, las clases de solfeo y piano se impartían de lunes a sábado, siempre en horario de tarde y tenían una duración de una hora y media (solfeo) y una hora (piano) (Sela, 1891, pp. 19, 20, 22).

La asignatura de "Nociones de Música" de la Escuela Preparatoria, que era impartida por Amorós, combinaba los rudimentos del lenguaje musical con el aprendizaje de algunas sencillas canciones. Recordemos que el canto coral fue una parcela indispensable de la educación musical para los institucionistas (Sánchez de Andrés, 2009b, pp. 286-304). Aniceto Sela describía así la metodología seguida en estas clases de iniciación a la música:

La clase de Música de la Escuela Preparatoria no ha de limitarse [...] a la enseñanza árida y seca del solfeo, que cansa y disgusta a las niñas. En este curso han aprendido algunas cancioncitas fáciles, pasando después de cantarlas de memoria a la notación musical, que sólo así les interesa (Sela, 1890, p. $6)$.

La Memoria del curso 1890 a 1891 nos aporta otros interesantes datos sobre los métodos empleados en las clases de solfeo y piano. En la Escuela Preparatoria, en la de Cultura General y en la de Institutrices, Amorós utilizaba su método Elementos de Solfeo (ca. 1889-1891). Elementos de Solfeo, reeditado hasta en diez ocasiones, consta de cien lecciones en clave de sol. Sobresale en este método una preocupación especial por la medida y la entonación. Las lecciones, con tesituras comprendidas entre el $\mathrm{Do}^{3}$ y el $\mathrm{Fa}^{4}$, están escritas en la tonalidad de Do Mayor o la menor (Micó Terol, 2014, p. 213).

En la Escuela de Bellas Artes, los tres cursos de Solfeo los impartía Amorós con dos métodos: el ya aludido Elementos del Solfeo, de su autoría, y otro método de Segura (probablemente Roberto Segura Villalba, profesor de piano de Amorós). El primer curso de Piano de la Escuela de Bellas Artes lo daba Adelaida Alarcón Cárcel con el Método Elemental de Piano de 
Roberto Segura (1879). Los cursos segundo y tercero de Piano los impartía Amorós con los métodos de Czerny y Cramer. La Memoria del curso 1890 a 1891 no especifica qué métodos de estos autores se utilizaron (Sela, 1891, pp. 19, 20, 22).

La Biblioteca de la IEM conservaba ejemplares de obras y métodos de piano y solfeo que habían sido adquiridos por compra o bien donados por los propios profesores. En 1888, la Biblioteca compró, por ejemplo, el Método de piano, ( $1^{\mathrm{a}}$ y $2^{\mathrm{a}}$ parte), de Czerny; el Método de Solfeo de Eslava; el Método de piano ( $1^{\mathrm{a}}$ y $2^{\mathrm{a}}$ parte) de Segura y Sonatinas (Un cuaderno), de Clementi (Sela, 1889b, pp. 19-21).

\section{Otras Actividades Musicales}

La IEM no sólo incorporó las asignaturas de Solfeo, Piano y Canto en su programa educativo sino que la música también estuvo presente, de manera destacada, en los actos de inauguración de sus cursos académicos, en las conferencias dominicales organizadas para señoras y en otros eventos como exposiciones y conmemoraciones diversas.

El repertorio que amenizaba los actos de inauguración, a cargo de músicos del círculo Goñi durante los primeros años de existencia de la IEM, supuso una auténtica promoción de la música de cámara con obras de Haydn, Mozart, Mendelssohn y Schumann (El Mercantil Valenciano, 1890, p. 2; El Mercantil Valenciano, 1892, p. 1). Junto a la música de cámara de origen germano, también se impulsó la interpretación de obras de compositores españoles. El programa que se ofreció en noviembre de 1888, dirigido por Amancio Amorós, incluyó la Serenata para quinteto de cuerda, piano y armónium, de Pedrell; L'ultimo addio, de Giner; la Canción de cuna, de Schumann; el Scherzo, de Plasencia, y la serenata de Antonio López Almagro, Non ti destare (Ilustración Musical Hispanoamericana, 1888, p. 184; Las Provincias, 1888, p. 2). Además, se estimuló la creación de composiciones de autores locales, como el coro "El Príncipe" de Manuel Palau, estrenado en una velada celebrada en homenaje a María Carbonell Sánchez el 1 de abril de 1928 (Las Provincias, 1928, p. 2).

En estas ceremonias solían participar las alumnas de Piano, Canto y Solfeo. El programa de la velada literario musical para conmemorar el tercer centenario de la publicación del Quijote (06-05-1905) consistió en: 
Mariani: "Al pie de la reja", serenata para piano, ejecutada por la niña Marcelina Luis.

"Las mujeres del Quijote", discurso por la profesora de la Escuela Normal de Maestras y de esta Institución, doña María Carbonell.

Weber: "Rondó Brillante", para piano, por la señorita Pepita Martín.

Lectura de un trozo del capítulo XIV, parte $1^{\mathrm{a}}$ del Quijote, por la alumna Desamparados Galán.

Mascagni: "Romanza de Santuzza", de la ópera Cavalleria Rusticana, cantada por la señorita Francisca García.

Lectura de la poesía del capítulo XLVI, parte $2^{\mathrm{a}}$ de la mencionada obra de Cervantes, por la alumna de la Escuela primaria, Dolores Vidal.

Raff: "Cachoucha", capricho para piano, por la alumna señorita García. Lectura por la señorita Llopis, alumna de la Escuela preparatoria, del capítulo $1^{\circ}$, parte $2^{\mathrm{a}}$ del Quijote.

Varvaró: "Messagiero d'amor", vals, cantado por la alumna señorita García.

Lectura del capítulo $\mathrm{V}$, parte $2^{\mathrm{a}}$ de la citada obra, por la alumna señorita Desamparados Gómez.

Giner: "Coro", cantado por las alumnas de la clase de solfeo (Las Provincias, 1905, p. 2. Véanse más ejemplos en: La correspondencia de Valencia, 1907, p. 2; Las Provincias, 1912, p.1).

En la ceremonia anual de entrega de premios a las alumnas más distinguidas del año 1932 las estudiantes Concha Sáiz, Ana López y Teresa Gómez interpretaron al piano obras de Diabelli, Chopin y Saint-Saens (Las Provincias, 1932, p. 12).

\section{Conclusiones}

Este estudio ha analizado el papel de la música en la Institución para la Enseñanza de la Mujer de Valencia desde su creación en 1888 hasta su extinción tras la Guerra Civil. A pesar de que existían centros educativos y academias particulares en las que se ofrecía educación musical, la IEM supuso un importante avance en materia de educación musical para la mujer 
valenciana, en sintonía con los preceptos krauso-institucionistas que concedían un puesto relevante a la música dentro de la educación.

El novedoso sistema educativo de la IEM contempló asignaturas de música (Solfeo, Piano y Canto) en casi todas sus escuelas, desde las etapas más tempranas hasta las enseñanzas profesionales. Al integrarla en la enseñanza general en todos los niveles educativos, los institucionistas no solo realzaron la música como una materia de cultura general, sino como parte de la formación integral de la mujer, perdiendo entonces el carácter complementario o "de adorno" que la música tenía, en general, en la época.

Ante el pobre panorama educativo valenciano, caracterizado por el insuficiente número de escuelas, la actitud de indiferencia y rechazo hacia la obligatoriedad de la escolarización y en general, la baja calidad de la enseñanza, la IEM contribuyó a consolidar un ambiente de renovación pedagógica desde una perspectiva progresista y laica, de acuerdo con los criterios renovadores que abogaban por la regeneración social a través de la enseñanza. La Institución que hemos estudiado fue un magnífico ejemplo del empeño de un grupo de intelectuales del siglo XIX por elevar la condición de la mujer y lograr su emancipación.

Por su prolongada trayectoria en el tiempo, así como por la importancia y el prestigio que alcanzó en la época contemporánea, la IEM merece un lugar destacado en la historia de la educación musical valenciana.

\section{Notas}

${ }^{1}$ Para el caso de Valencia, véase Mínguez Blasco, 2011. Sobre el panorama educativo valenciano en el siglo XIX y principios del XX, consúltese Agulló Díaz, 1990; Martínez Bonafé, 1985; Piqueras Arena, 1980, 1983.

${ }^{2}$ En diversos estudios sobre música valenciana de esta época se aportan datos de interés sobre centros educativos y otras instituciones en las que se impartían clases de música (Fontestad, 2011, pp. 23-27; Galbis López, 1999, pp. 86-87).

${ }^{3}$ Los distintos discursos pronunciados en la inauguración de cada curso académico, y en otras ocasiones, son fuentes de inestimable valor para conocer el ideario de esta Institución. Ver, 
HSE - Social and Education History, 8(1) 49

Serrano, 1888; Oliver, 1887, 1923; Sela, 1888b; Pardo de la Casta, 1889; Boscá y Casanoves, 1891 y Sampil de Sela, 1892.

\section{Fuentes}

\section{Archivo Particular de Rafael Solaz Albert}

Fotografía "Alumnas recibiendo clase de Dibujo en el Colegio Santa Ana de Valencia (1913)".

\section{Archivo de la Real Sociedad Económica de Amigos del País de Valencia (E:VAseap)}

C. 222, III-Educación n ${ }^{\circ}$ 11, "Documentación relativa a la creación de la Escuela de Comercio para Señoras (1883)". Incluye manuscrito del Reglamento de la Escuela de Comercio para Señoras (1883)

C. 246, X-Contabilidad no 11 , "Libramiento de pago de ciertas cantidades en concepto de subvenciones para el Conservatorio de Música y Escuela de Comercio (1888)"

C. 255, III-Educación $n^{\circ} 8$, "Folleto relativo al espíritu que anima y al funcionamiento de la Institución para la Enseñanza de la Mujer (1891)"

C. 258, III-Educación $\mathrm{n}^{\circ}$ 1, "Documentación relativa a la constitución de la Institución de la Enseñanza de la Mujer y su Reglamento (1892)"

\section{Archivo Histórico Municipal de Valencia (E:Vaa)}

Sección III, Subgrupo G Instrucción Pública, Clase II (Enseñanza Superior)

Subclase C, Escuelas Especiales (1850-1900), n 1 1, "La Sociedad

Económica de Amigos del País, solicita una subvención para establecer en esta capital una escuela de comercio para señoras (1883)" 
Sección III, Subgrupo G Instrucción Pública, Clase II (Enseñanza Superior) Subclase C, Escuelas Especiales (1850-1900), n 1, "La sociedad de Amigos del País solicita la subvención de 2000 pesetas en favor de la escuela de comercio para señoras (1886)"

\section{Referencias hemerográficas}

Ilustración Musical Hispanoamericana, I (1888), nº 23, 30 diciembre, p. 184 El Mercantil Valenciano, 19 (1890), no 7825, 16 noviembre, p. 2

El Mercantil Valenciano, 21 (1892), $\mathrm{n}^{\circ} 8539,31$ octubre, p. 1

La Correspondencia de España, XLVIII (1925), n 19739, 23 de septiembre, p. 2

La Correspondencia de Valencia, XXX (1907), $\mathrm{n}^{\circ} 10098,3$ mayo, p. 2

La Correspondencia de Valencia, XL (1917), n 17198, 24 septiembre, p. 2

La Correspondencia de Valencia, LVI (1933), no 22220, 14 septiembre, p. 2 Las Provincias, 23 (1888), $\mathrm{n}^{\circ}$ 8122, 18 noviembre, p. 2

Las Provincias, XXXVIII (1903a), no 13425, 30 mayo, p. 2

Las Provincias, XXXVIII (1903b), no 13427, 1 junio, p. 2

Las Provincias, XXXVIII (1903c), no 13432, 6 junio, p. 2

Las Provincias, XL, 1905, $\mathrm{n}^{\circ}$ 14130, 6 mayo, p. 2

Las Provincias, XLVII (1912), no 16732,19 julio, p. 1

Las Provincias, 58 (1923), n ${ }^{\circ} 16588,11$ febrero, p. 4

Las Provincias, 63 (1928), $\mathrm{n}^{\circ}$ 19265, 30 marzo, p. 2

Las Provincias, 67 (1932), n 20524, 22 julio, p. 12

\section{Referencias Bibiliográficas}

Agulló Díaz, C. (1990). Mujeres para Dios, para la Patria y para el Hogar. En Mujer y Educación en España 1868-1975, IV Coloquio de Historia de la Educación. Santiago de Compostela: Universidad de Santiago, 17-26.

Agulló Díaz, C. (2011). Participación política, renovación pedagógica e independencia personal: El triple compromiso de las maestras republicanas valencianas. En Barrio Alonso, A., Hoyos Puente, J. de, Saavedra Arias, R., Nuevos horizontes del pasado. Culturas políticas, 
identidades y formas de representación: Actas del X Congreso de la Asociación de Historia Contemporánea, 38.

Alarcón, J. [1890]. Discurso leído en el acto de apertura del Curso de 1890

a 1891 de la Institución para la Enseñanza de la Mujer. Valencia.

Alcázar Cruz Rodríguez, M. (2007). El acceso de las mujeres a la educación como eje fundamental para su promoción y participación. Sumuntán: anuario de estudios sobre Sierra Mágina, 24, 9-30.

Ballarín, P. (1989). La educación de la mujer española en el siglo XIX.

Historia de la Educación: Revista interuniversitaria, 8, 245-260.

Blasco Carrascosa, J. A. (1980). Un arquetipo pedagógico pequeño-burgués.

(Teoría y praxis de la Institución Libre de Enseñanza). Valencia:

Fernando Torres.

Blasco Carrascosa, J. A. (1982). Krausisme i Renaixença a València.

Valencia: Fernando Torres, editor.

Blasco Carrascosa, J. A. (1984). El krausisme valencià. València: Institució Alfons el Magnànim.

Boscá y Casanoves, Eduardo (1891). Discurso inaugural del año académico de 1891-92. Valencia: Imprenta de Francisco Vives Mora.

Bueno Ortuño, J. (1979). La Institución Libre de Enseñanza y Valencia.

Valencia: Real Sociedad Económica de Amigos del País.

Canes Garrido, F. (1990). La educación de la mujer en las memorias técnicas

de las maestras de las escuelas públicas de la Ciudad de Valencia

(1908-1909). En Mujer y Educación en España 1868-1975, IV

Coloquio de Historia de la Educación. Santiago de Compostela:

Universidad de Santiago, 87-94.

Cantizano Márquez, B. (2004). La mujer en la prensa femenina del siglo XIX. Ámbitos, 2, 281-298.

Clares-Clares, Esperanza (2017). Música y noches de moda. Sociedades,

cafés y salones domésticos de Murcia en el siglo XIX. Murcia:

Universidad de Murcia.

Delgado Criado, B. (coord.) (1994). Historia de la educación en España y

América. La educación en la España contemporánea (1879-1975).

Madrid: Ediciones SM, vol. 3.

Escuela de Comercio (1884). Reglamento de la Escuela de Comercio para

Señoras. Valencia: Imprenta de Nicasio Rius. 
Esteban Mateo, L. (1974). La Institución libre de Enseñanza en Valencia. Valencia: Donaire.

Fontestad, A. (2011). El Conservatorio de Música de Valencia. Precedentes e historia temprana (1850-1910). Valencia: Institut Valencià de la Música.

Galbis López, V. (1999). La educación musical española en el siglo XIX: el caso valenciano. Eufonía, 17, 79-87. Recuperado de http://visor.grao.com/?params=eyJET0MiOiIwMDU0OTMyOS54bW wiLCJET1dOTE9BRCI6Imh0dHBzOlwvXC93d3cuZ3Jhby5jb21cL2 NhXC9wcm9kdWN0ZXNcL2Rlc2Nhcmdhci1wZGY_c2x1Zz1sYS11 ZHVjYWNpb24tbXVzaWNhbC1lc3Bhbm9sYS1lbi1lbC1zaWdsby14 aXgtZWwtY2Fzby12YWxlbmNpYW5vIiwiSVAiOiIqIiwih

Institución para la Enseñanza de la Mujer (IEM) (1889). Prospecto para el curso 1889-1890. Valencia.

Institución para la Enseñanza de la Mujer (IEM) (1890). Reglamento de la Institución para la Enseñanza de la Mujer. Valencia: Imprenta de Francisco Vives Mora.

Institución para la Enseñanza de la Mujer (IEM) [1891]. Prospecto para el curso de 1891-1892. Valencia: Imprenta Vives.

Institución para la Enseñanza de la Mujer (IEM) [1893]. Prospecto para el Curso 1893-1894. Valencia: Imprenta Gay.

Institución para la Enseñanza de la Mujer (IEM) (1895). Institución para la Enseñanza de la Mujer. Almanaque de las Provincias para 1895, 279284.

Institución para la Enseñanza de la Mujer (IEM) [1896]. Prospecto para el curso 1896-1897. Valencia: Imprenta y Litografía de José Ortega. Institución para la Enseñanza de la Mujer (IEM) (1899). Prospecto para el curso 1899-1900. Valencia.

Institución para la Enseñanza de la Mujer (IEM) (1915). Asociación de Profesoras y Alumnas de la Institución para la Enseñanza de la Mujer. Reglamento. Valencia: La Gutenberg.

Lázaro Lorente, L. M. (1992). Las escuelas racionalistas en el País Valenciano (1906-1931). Valencia: Nau Llibres.

Mandado Gutiérrez, R. E., Sánchez-Gey Venegas, J. y Madariaga de la Campa, B. (coords.) (2011). La Institución Libre de Enseñanza y la Asociación para la Enseñanza de la Mujer: bosquejo sobre la 
educación española del siglo XIX. Santander: Universidad Internacional Menéndez Pelayo.

Marín Silvestre, Dolors (2018). Espiritistes i lliurepensadores: dones pioneres en la lluita pels drets civils. Barcelona: Angle Editorial, 2018.

Martínez Bonafé, A. (1985). Ensenyament, burgesia i liberalisme. València: Diputació Provincial de València.

Micó Terol, E. (2014). El compositor Amancio Amorós Sirvent (1854-1925) en el contexto musical de Valencia. Lleida: Fundació Pública Institut d'Estudis Ilerdencs de la Diputació de Lleida.

Mínguez Blasco, R. (2011). La educación de las mujeres en la ciudad de Valencia antes de la ley Moyano. En Barrio Alonso, A., Hoyos Puente, J. de, Saavedra Arias, R., Nuevos horizontes del pasado. Culturas políticas, identidades y formas de representación: Actas del X Congreso de la Asociación de Historia Contemporánea, 40.

Oliver, J. A. (1887). De la necesidad de la instrucción en la mujer para que pueda realizar su misión sobre la tierra. Valencia: Imprenta Unión Tipográfica.

Oliver, J. A. (1892). Memoria del curso de 1891 a 1892. Institución para la Enseñanza de la mujer. Valencia: Imprenta de Francisco Vives Mora. Oliver, J. A. (1913). La educación de la mujer en Valencia. Valencia:

Manuel Pau.

Oliver, J. A. (1923). La importancia del feminismo. Valencia: Talleres Tipográficos "Las Artes".

Ontañón Sánchez, E. (2003). Un estudio sobre la institución libre de enseñanza y la mujer. Valencia: Universidad Politécnica de Valencia. Palà Moncusí, Albert (2018). Viure l'anticlericalisme: una història cultural del lliure pensament català (1868-1923). Barcelona Editorial Afers.

Palacio, I. (1992). Mujer, Trabajo y Educación (Valencia 1874-1931).

Valencia: Departamento de Educación Comparada e Historia de la Educación, Universitat de València.

Pardo de la Casta, J. (1889). La mujer en el siglo XIX. Valencia: 1889. Parreño Arenas, E. (2011). Mujer y Educación. Una mirada sobre la educación femenina durante el siglo XIX, III Congreso virtual sobre Historia de las mujeres del 15 al 31 de octubre de 2011. Jaén: 
Asociación de Amigos del Archivo Histórico Provincial. Recuperado de http.//www.revistacodice.es/publi_virtuales/iii_congreso_mujeres/ Piqueras Arena, J. (1980). Educación y crecimiento burgués en el País Valenciano. Anales del Centro de Alzira de la Universidad Nacional de Educación a Distancia, 1, 373-391.

Piqueras Arena, J. (1983). El taller y la Escuela en la Valencia del siglo XIX. Valencia: Ayuntamiento de Valencia.

Pinedo Herrero, C., Más Zurita, E. y Mocholí Roselló, A. (2003). 250 años, la enseñanza de las Bellas Artes en Valencia y su repercusión social.

Valencia: Facultad de Bellas Artes, Universidad Politécnica de Valencia.

Ramos López, P. (2003). Feminismo y música: introducción crítica. Madrid: Narcea, Fundación Invesnes.

Reig, A. (2012). El compromiso social de un político krausista. Rafael Albiñana y la Institución para la Enseñanza de la Mujer de Valencia. Valencia: Martín Impresores.

Sampil de Sela, María (1892). Discurso inaugural del año académico de 1892 a 1893. Valencia: Local de la Institución.

Sánchez de Andrés, L. (2005a). El pensamiento estético del Krausismo español. Revista de Musicología, 28/2, 961-976.

Sánchez de Andrés, L. (2005b). La música en la actividad educativa

institucionista. Boletín de la Institución Libre de Enseñanza, II Época, 57, 7-18.

Sánchez de Andrés, L. (2008). Compositoras españolas del siglo XIX: la lucha por espacios de libertad creativa desde el modelo de feminidad decimonónico. En Álvarez Cañibano, A. (coord.) (2008).

Compositoras españolas. La creación musical femenina desde la Edad Media hasta la actualidad. Madrid: Centro de Documentación de Música y Danza, 55-74.

Sánchez de Andrés, L. (2009a). El pensamiento y la actividad musical de Francisco Giner de los Ríos. Iniciativas krausoinstitucionistas en el ámbito de la educación musical (1869-1915). En Vázquez-Romero, J. M. (coord.) (2009). Francisco Giner de los Ríos. Actualidad de un pensador krausista. Madrid: Marcial Pons, 2009, 199-258. 
Sánchez de Andrés, L. (2009b). Música para un ideal. Pensamiento y actividad musical del krausismo e institucionismo españoles (18541936). Madrid: Sociedad Española de Musicología.

Sanfeliu, L. (2005). Republicanas: identidades de género en el blasquismo (1895-1910). València, Universitat de València.

Scanlon, G. (1987). La mujer y la instrucción pública: de la ley Moyano a la II República. Historia de la Educación, 6. Recuperado de http://revistas.usal.es/index.php/0212-0267/article/view/6743

Sela, A. (1888a). La enseñanza de la mujer en Valencia. Escuela de Comercio, Boletín de la Institución Libre de Enseñanza, 30 de abril, 109-111.

Sela, A. (1888b). Sobre la Educación física de la mujer. Valencia: Imprenta Unión Tipográfica.

Sela, A. (1889a). Una nueva Institución para la Enseñanza de la Mujer. Boletín de la Institución Libre de Enseñanza, 15 de diciembre, 353356.

Sela, A. [1889b]. Memoria del curso de 1888 a 1889. Institución para la Enseñanza de la mujer. Valencia: Imprenta de Francisco Vives y $\mathrm{C}^{\mathrm{a}}$.

Sela, A. [1890]. Memoria del curso de 1889 a 1890. Institución para la Enseñanza de la mujer. Valencia: Imprenta de Francisco Vives Mora.

Sela, A. [1891]. Memoria del curso de 1890 a 1891. Institución para la Enseñanza de la mujer. Valencia: Imprenta de Francisco Vives Mora.

Serrano, E. (1888). La Mujer instruida para el Comercio. Valencia: Imprenta Unión Tipográfica.

Sole Romero, G. (1990). La instrucción de la mujer en la Restauración. La Asociación para la Enseñanza de la mujer. Tesis Doctoral. Madrid: Facultad de Geografía e Historia, Universidad Complutense de Madrid.

Tahull, J., Montero, I., \& Molina, F. (2016). Segle XIX. Esperances i fracassos de la modernitat a l'educació. Situació d'Espanya. Social and Education History 5(1), 26-51. doi.10.17583/hse.2016.1718

Vázquez Ramil, R. (2001). La Institución Libre de Enseñanza y la educación de la mujer en España: la Residencia de Señoritas (19151935). Betanzos: Lugami. 
56 Clares-Clares \& Micó-Terol-La educación musical

Esperanza Clares-Clares: Universidad de Murcia

Elena Micó-Terol: IES Jaume I Ontinyent (Valencia) Generalitat Valenciana

Contact Address: eclares@um.es 